\title{
Transient behaviour in RDA systems of the Schnakenberg type
}

\author{
Aya Al-Zarka • Afnan Alagha $\cdot$ S. Timoshin
}

Received: 25 March 2014 / Accepted: 15 September 2014 / Published online: 15 October 2014 (C) The Author(s) 2014. This article is published with open access at Springerlink.com

\begin{abstract}
Initial stages in the evolution of linear disturbances near a homogeneous equilibrium are considered for the standard Schnakenberg and modified Schnakenberg models. The focus is on a possibility of transient amplification of perturbations. It is shown that, depending on the coefficients in the governing equations, transient growth may appear in both asymptotically stable and unstable situations.
\end{abstract}

Keywords Transient growth $\cdot$ Schnakenberg model $\cdot$ Linear instability

\section{Introduction}

Reaction-diffusion-advection (RDA) models typically exhibit several types of instability, depending on the particulars of the model and the parameters chosen to study the stability of equilibrium states (e.g. [1,5]). Along with the common traveling-wave instability, the Turing or diffusion-driven instability attracted much attention, starting with the pioneering investigation by Turing [13].

It was probably not until the work of Neubert et al. [6] that some emphasis began to emerge on the early-time behaviour of the RDA systems. It was found that under the conditions of Turing instability, the linearized kinetics matrix is reactive, that is initial disturbances are amplified at the start of the evolution. More recently, Elragig and Townley [2] found that the Turing instability is not possible if the reaction matrix and

\footnotetext{
A. Al-Zarka · A. Alagha

Nonlinear Analysis and Applied Mathematics Research Group (NAAM), Department of Mathematics, King Abdulaziz University, Jeddah, Saudi Arabia

S. Timoshin $(\bowtie)$

Department of Mathematics, UCL, London, UK

e-mail: sergei.timoshin@gmail.com
} 
the diffusion matrix share a common Lyapunov function. Addressing early system's evolution from a somewhat different angle, Ridolfi et al. [8] discuss the emergence of non-trivial statistical properties in a Turing system due to transient modes.

The aim in this study is to clarify the role of transient modes in the evolution of linear disturbances governed by Schankenberg-type kinetics. The Schankenberg [9] model has received a great deal of attention, see e.g. [3-5,7]. In this study, the Schnakenberg model will be re-examined first from the point of view of modal stability but paying more attention than usual to the properties sometimes associated with transient behaviour. We then show how the linear kinetics matrix can be extended to provide conditions for enhanced transient growth. Examples of wave-packet evolution initiated by a localized smooth initial perturbation are given, showing transient growth in both asymptotically stable and unstable regimes.

\section{The Schnakenberg model: linear modes}

The Schnakenberg model, with a constant advection term added, is governed by the equations,

$$
\begin{aligned}
& \frac{\partial u}{\partial t}+c \frac{\partial u}{\partial x}=a-u+u^{2} v+\mu_{1} \nabla^{2} u \\
& \frac{\partial v}{\partial t}+c \frac{\partial v}{\partial x}=b-u^{2} v+\mu_{2} \nabla^{2} v
\end{aligned}
$$

where $\nabla^{2}=\partial^{2} / \partial x^{2}+\partial^{2} / \partial y^{2}$. It is assumed conventionally that the parameters are non-negative, $a>0, b>0, c \geq 0$; also the diffusion coefficients are necessarily positive.

The equilibrium, homogeneous, states of the Schnakenberg system are given by

$$
u_{0}=a+b, \quad v_{0}=\frac{b}{(a+b)^{2}} .
$$

Considering small perturbations about the base uniform state, we write, $u=u_{0}+$ $\varepsilon u_{1}$

$+\cdots, v=v_{0}+\varepsilon v_{1}+\cdots$, which gives a system of linearized equations,

$$
\begin{aligned}
& \frac{\partial u_{1}}{\partial t}+c \frac{\partial u_{1}}{\partial x}=\left(q_{1}-1\right) u_{1}+q_{2} v_{1}+\mu_{1} \nabla^{2} u_{1}, \\
& \frac{\partial v_{1}}{\partial t}+c \frac{\partial v_{1}}{\partial x}=-q_{1} u_{1}-q_{2} v_{1}+\mu_{2} \nabla^{2} v_{1},
\end{aligned}
$$

with the coefficients $q_{1}=2 b /(a+b), q_{2}=(a+b)^{2}$.

2.1 One spatial dimension

If we restrict ourselves to just one spatial dimension, the system simplifies further, 


$$
\begin{aligned}
& \frac{\partial u_{1}}{\partial t}+c \frac{\partial u_{1}}{\partial x}=\left(q_{1}-1\right) u_{1}+q_{2} v_{1}+\mu_{1} \frac{\partial^{2} u_{1}}{\partial x^{2}}, \\
& \frac{\partial v_{1}}{\partial t}+c \frac{\partial v_{1}}{\partial x}=-q_{1} u_{1}-q_{2} v_{1}+\mu_{2} \frac{\partial^{2} v_{1}}{\partial x^{2}} .
\end{aligned}
$$

To begin with, the disturbance in the form of a single spatially periodic mode can be taken, so that

$$
\left(\begin{array}{l}
u_{1} \\
v_{1}
\end{array}\right)=e^{\lambda t+i k x}\left(\begin{array}{l}
U \\
V
\end{array}\right),
$$

leading to the eigenvalue problem,

$$
\lambda\left(\begin{array}{l}
U \\
V
\end{array}\right)=A\left(\begin{array}{l}
U \\
V
\end{array}\right)
$$

with the matrix

$$
A=\left(\begin{array}{cc}
-c i k+q_{1}-1-\mu_{1} k^{2} & q_{2} \\
-q_{1} & -c i k-q_{2}-\mu_{2} k^{2}
\end{array}\right) .
$$

\subsection{Eigenmodes of the linear Schnakenberg model}

In the modal analysis the first straightforward question to addres is the stability of the linear modes, as determined by the sign of the real part of the eigenvalues given by

$$
\lambda_{ \pm}=\frac{1}{2} \operatorname{Tr} A \pm \frac{1}{2} \sqrt{\operatorname{Tr}^{2} A-4 \operatorname{det} A},
$$

in terms of the trace and the determinant of $A$. A representative example of the growth rates of the linear modes for long waves is shown in Fig. 1.

The real part of the eigenvalue with the minus sign is shown in Fig. 2, whereas Fig. 3 demonstrates the non-negative imaginary part of the eigenvalues when the eigenvalues are complex valued.

It is clear from these diagrams that instability in the linear Schnakenberg system without advection appears as an oscillatory mode (with complex-valued growth rates) or, alternatively, as a Turing mode with purely real eigenvalues.

As shown in Fig. 4, the effect of a non-zero wavenumber is to reduce the growth rate due to dissipation.

Instability is related to elevated levels of the condition number for the matrix of the right-hand side in the single-mode equations, as shown in Fig. 5.

\subsection{Non-normal eigenvectors}

The eigenvectors of (2.10) are in general not orthogonal. As a measure of nonnormality of the matrix one can take a level of non-orthogonality of the eigenvectors. 


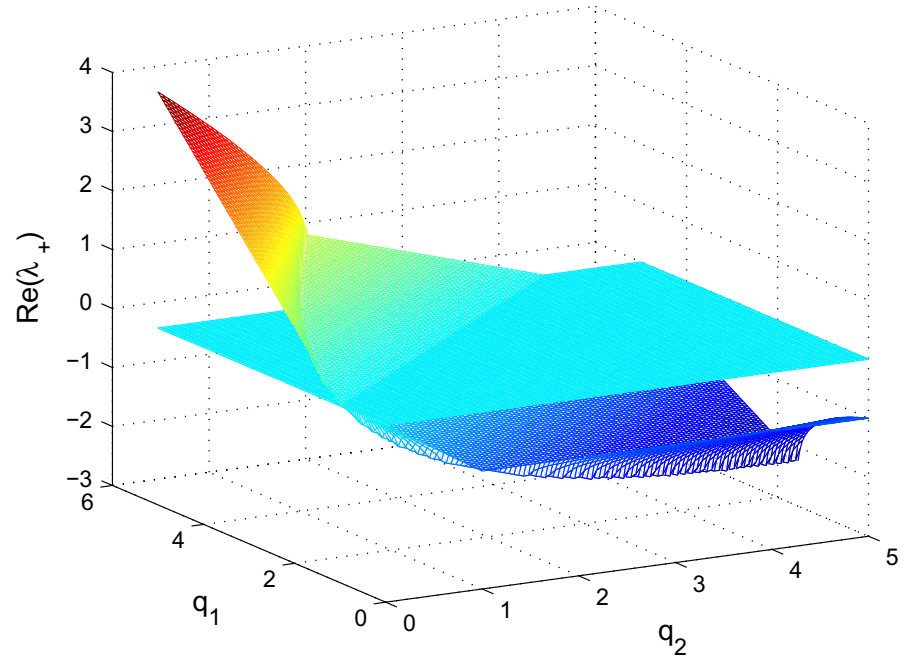

Fig. 1 Real part of the eigenvalue $\lambda_{+}$for $k=0, \mu_{1}=1, \mu_{2}=5, c=0$, against the parameters $q_{1}, q_{2}$

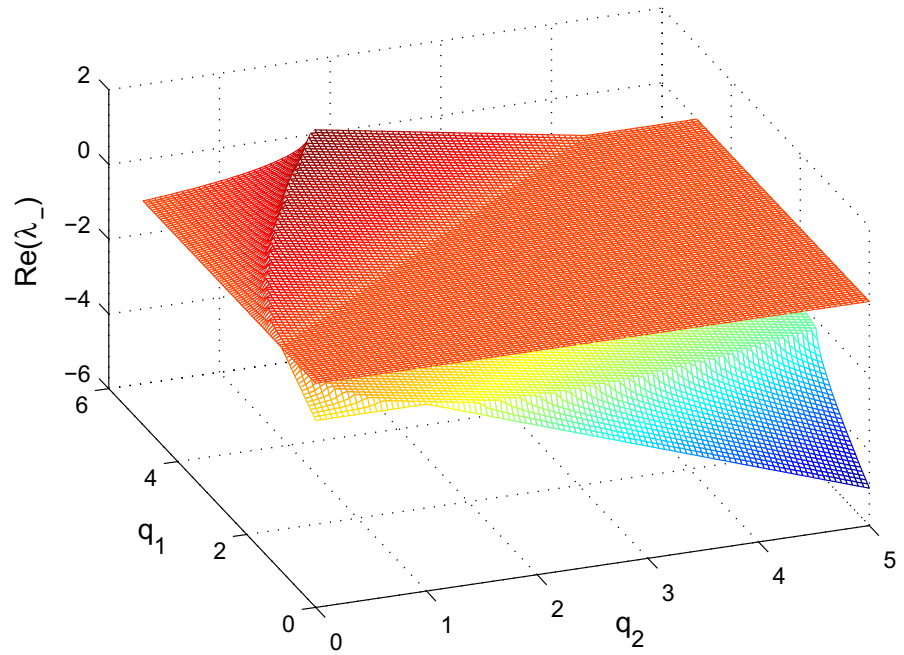

Fig. 2 The real part of the second eigenvalue for the same parameters

For the Schnakenberg system, in the extreme case the eigenvectors can even be parallel. Indeed, the eigenvalues given by the formula (2.11) have the associated eigenvectors,

$$
\left(\begin{array}{c}
U_{ \pm} \\
V_{ \pm}
\end{array}\right)=\left(\begin{array}{c}
1 \\
-q_{2}^{-1}\left(q_{1}-1-\mu_{1} k^{2}-\lambda_{ \pm}\right)
\end{array}\right) .
$$

The eigenvectors are parallel if $\lambda_{+}=\lambda_{-}$which happens when $(\operatorname{Tr} A)^{2}=4 \operatorname{det} A$ or

$$
\left(\sqrt{q_{1}}-\sqrt{q_{2}}\right)^{2}=1+\left(\mu_{1}-\mu_{2}\right) k^{2} .
$$




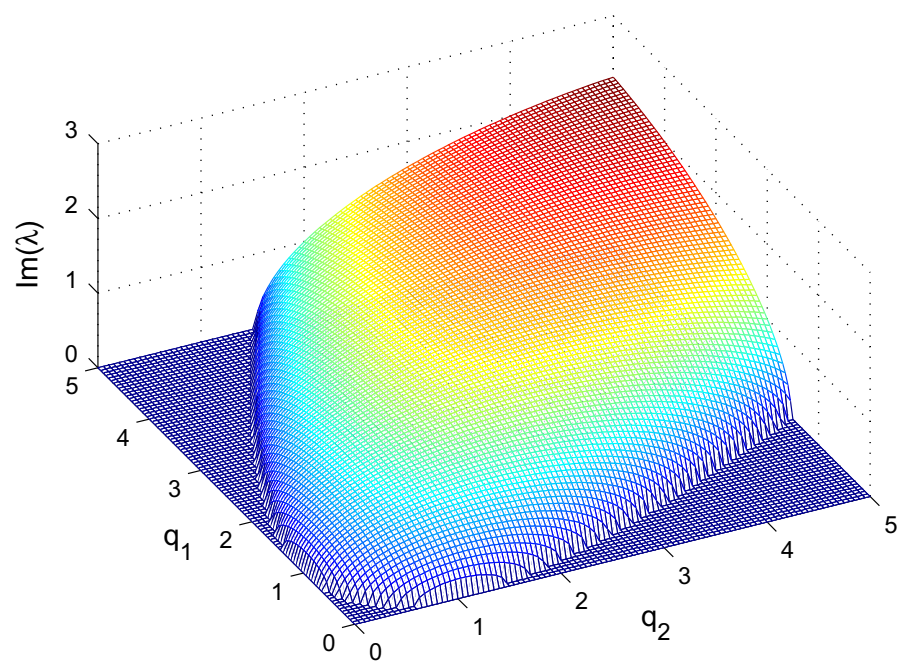

Fig. 3 The imaginary part for the eigenvalues

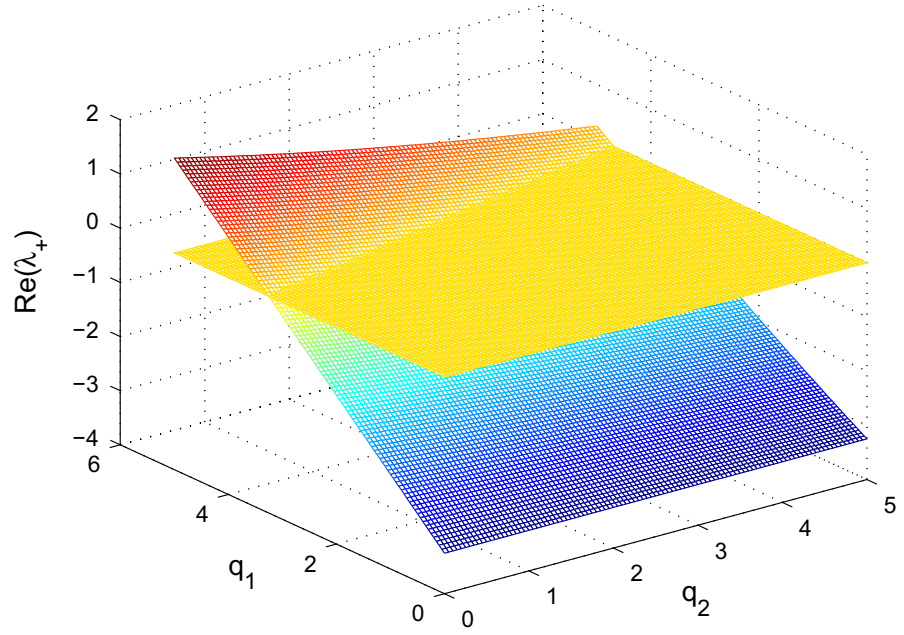

Fig. 4 The growth rate of a mode with a non-zero wavenumber, $k=1.5$, with the other parameters unchanged

For given parameters of the system, this equation determines the wavenumber $k$ for the disturbance. However, it turns out that alternative characteristics, in particular a condition number of the matrix, is a more practical tool in investigations of transient growth.

\subsection{The Turing space}

In general, we cannot expect any non-oscillatory growing mode to be a Turing mode. The conditions of Turing instability (understood as diffusion driven instability in a 


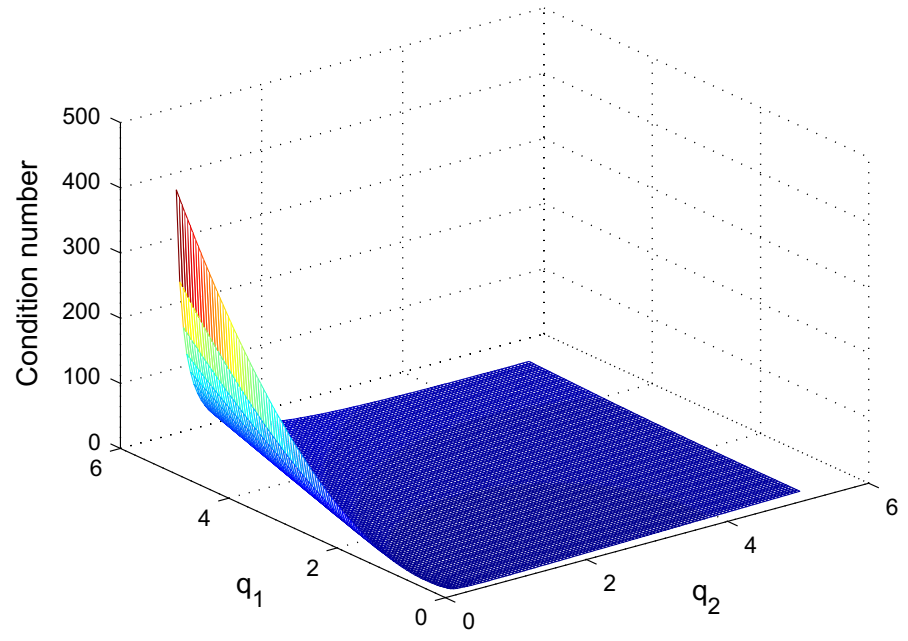

Fig. 5 The condition number of the linearized system for long waves $|k| \ll 1$

stable reactive system) are very restrictive. In our notation, the Turing space is specified by the inequalities,

$$
\begin{aligned}
& q_{1}<1+q_{2}, \\
& q_{2}>0, \\
& \mu_{2}\left(q_{1}-1\right)-\mu_{1} q_{2}>0, \\
& q_{1}>\left(1+\sqrt{\frac{\mu_{1}}{\mu_{2}} q_{2}}\right)^{2} .
\end{aligned}
$$

In the $(a, b)$-parameter plane, the Turing instability region appears as a narrow wedge, as shown in Fig. 6.

\subsection{Modal behaviour}

The behavior of a single mode with a wavenumber $k$ can be investigated as a solution of Eqs. (2.6)-(2.7) with the initial conditions,

$$
u_{1}(x, t)=u_{10} \cos (k x), v_{1}(x, t)=v_{10} \cos (k x) .
$$

The solution is then written as

$$
\begin{aligned}
& u_{1}=e^{i k x} \tilde{u}_{1}(t)+c . c . \\
& v_{1}=e^{i k x} \tilde{v}_{1}(t)+c . c .
\end{aligned}
$$

and the time-dependent parts of the solution, $\tilde{u}_{1}(t), \tilde{v}_{1}(t)$ are found in terms of the eigenfunctions (2.8) as 


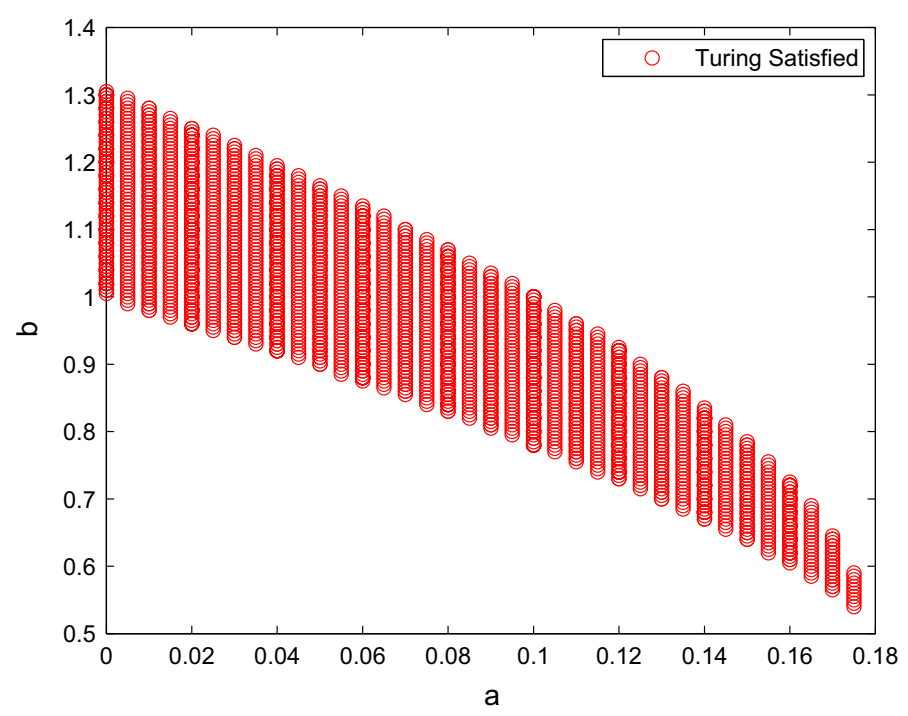

Fig. 6 The Turing space in the $(a, b)$-plane for $c=0, \mu_{1}=1, \mu_{2}=10$

$$
\left(\begin{array}{c}
\tilde{u}_{1} \\
\tilde{v}_{1}
\end{array}\right)=C_{1} e^{\lambda_{+} t}\left(\begin{array}{c}
U_{+} \\
V_{+}
\end{array}\right)+C_{2} e^{\lambda_{-} t}\left(\begin{array}{c}
U_{-} \\
V_{-}
\end{array}\right) .
$$

The constants $C_{+}, C_{-}$are determined by expressing the vector of initial conditions in terms of the eigenvectors ("Appendix").

As a measure of the disturbance magnitude for a specified wavenumber $k$ we evaluate the solution at $x=0$ and take

$$
N(t)=\frac{\sqrt{u_{1}^{2}+v_{1}^{2}}}{\sqrt{u_{10}^{2}+v_{10}^{2}}} .
$$

When the interest is in individual components of the solution, we adopt the normalization,

$$
u_{1 N} \rightarrow \frac{u_{1}}{\sqrt{u_{10}^{2}+v_{10}^{2}}}, \quad v_{1 N}=\frac{v_{1}}{\sqrt{u_{10}^{2}+v_{10}^{2}}} .
$$

In general, the solution can display either monotonic or transient behaviour depending on the parameters of the model. In Fig. 7 we have an example of transient oscillations in a stable range of parameters. However, the notion of transients requires some care. The following Fig. 8 shows that the total norm of the solution may be strictly monotonic (decaying in this case) when individual components of the solution exhibit oscillatory transients.

In Fig. 9 we observe oscillatory instability. However, a wave with a different wavelength may be unstable monotonically for the same system parameters, as seen in Fig. 10. 


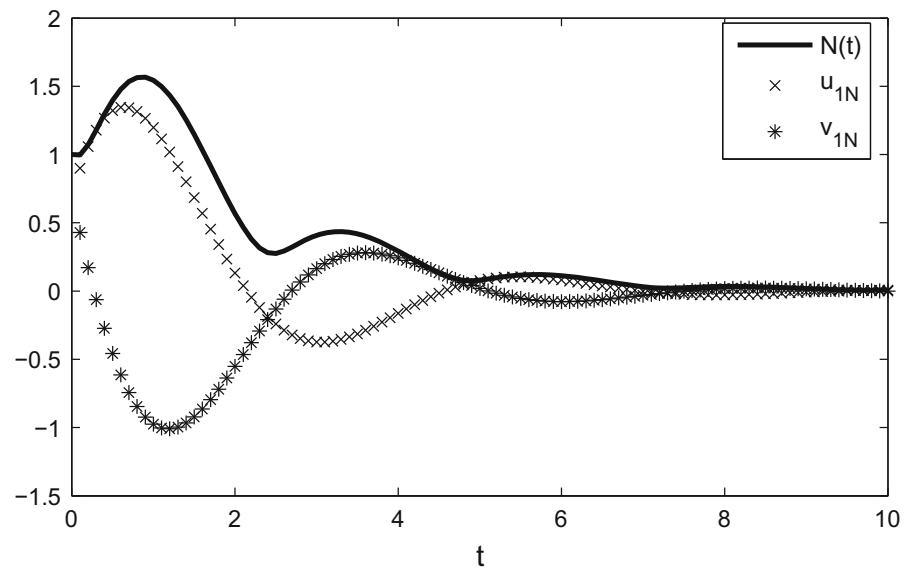

Fig. 7 Single mode solution with $q_{1}=q_{2}=2, k=0.1, c=0, \mu_{1}=1, \mu_{2}=5$

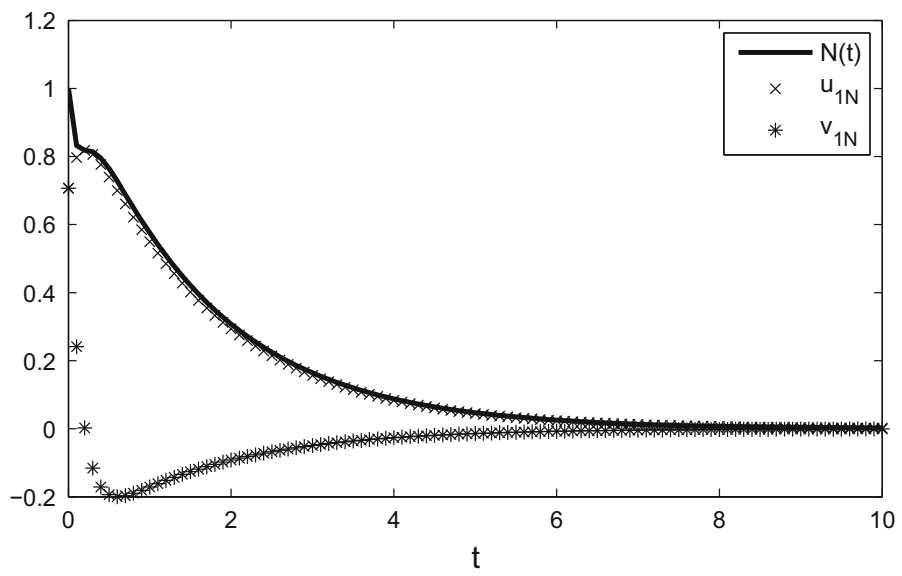

Fig. 8 Same as in Fig. 7 but with the wavenumber $k=1$

\section{Modified Schnakenberg model}

In order to investigate further possible transient effects in RDA systems, we modify the original linearized Schnakenberg model and include a higher level of asymmetry in the $v_{1}$ term in the reactive part. The system now reads,

$$
\begin{aligned}
& \frac{\partial u_{1}}{\partial t}+c \frac{\partial u_{1}}{\partial x}=\left(q_{1}-1\right) u_{1}+\left(q_{2}+s\right) v_{1}+\mu_{1} \frac{\partial^{2} u_{1}}{\partial x^{2}} \\
& \frac{\partial v_{1}}{\partial t}+c \frac{\partial v_{1}}{\partial x}=-q_{1} u_{1}-q_{2} v_{1}+\mu_{2} \frac{\partial^{2} v_{1}}{\partial x^{2}}
\end{aligned}
$$

in the case of one spatial dimension. The matrix (2.10) is replaced by 


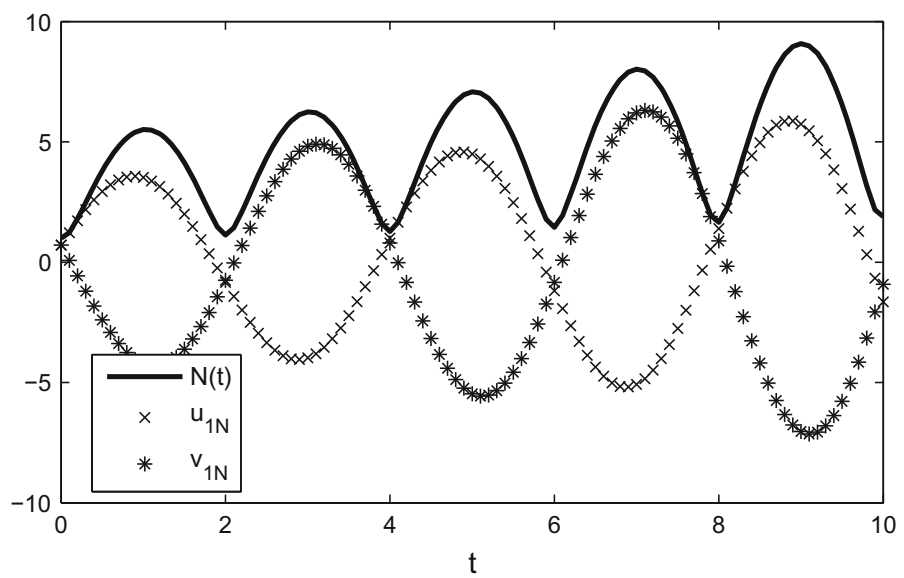

Fig. 9 Oscillatory instability; $q_{1}=5, q_{2}=3.5, k=0.25, c=0, \mu_{1}=1, \mu_{2}=5$

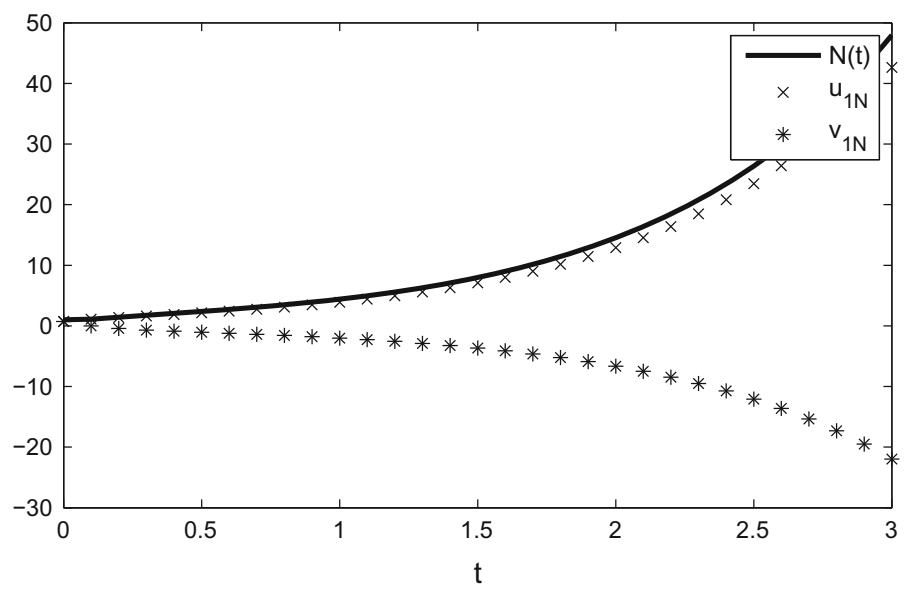

Fig. 10 Same as in Fig. 9 but with $k=1$

$$
A=\left(\begin{array}{ll}
-c i k+q_{1}-1-\mu_{1} k^{2} & q_{2}+s \\
-q_{1} & -c i k-q_{2}-\mu_{2} k^{2}
\end{array}\right) .
$$

The appearance of an additional term $s v_{1}$ in the Eq. (3.1) shifts the total balance in the system away from a $u$-dominated dynamics to more evenly distributed interaction between the two reacting components (as can be seen considering the sum of the two equations, for example). Loosely speaking, the amount of this shift is measured by the magnitude of $s$. The aim in this section will be to investigate the effect of the extra term on the system behaviour.

The diagrams in Figs. 11 and 12 illustrate rather dramatic changes in the distribution of the condition number in the plain of the parameters $\left(q_{1}, q_{2}\right)$ as the shift constant, $s$, increases to $s=1$ and $s=10$. 


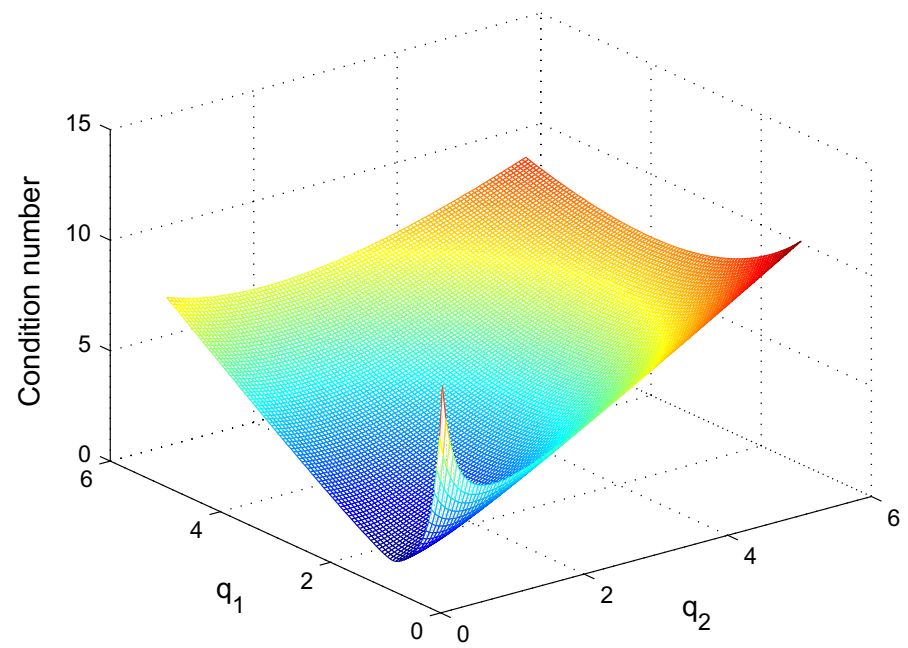

Fig. 11 The condition number for long waves $|k| \ll 1$ at $s=1$; the other parameters are $\mu_{1}=1$, $\mu_{2}=5, c=0$

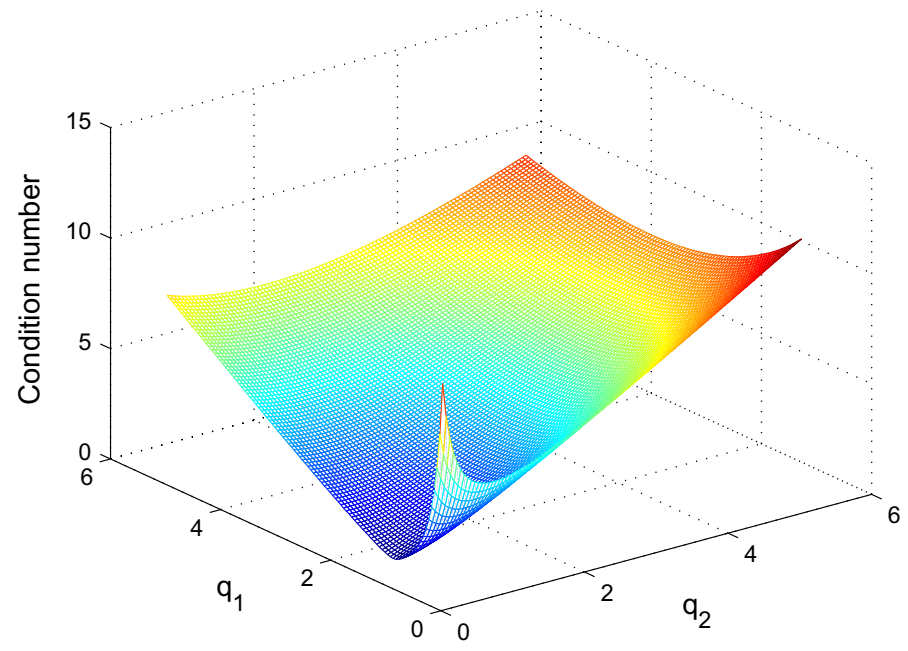

Fig. 12 As in Fig. 11 but with $s=10$

It is clear that at larger values of $s$ a region of interest emerges at small values of $q_{1}$ and $q_{2}$. This is consistent with intuitive appreciation of large values of $s$ as having a greater influence on the normality of the eigenvectors of the system even for sufficiently small values of $q_{2}$.

In the majority of this section we focus on relatively large $s$ and moderately small $q_{1}, q_{2}$ where we expect the system to be stable. First we consider a series of computations performed with the initial conditions,

$$
u_{1}=e^{-x^{2}}, \quad v_{1}=e^{-x^{2}} \text { at } t=0,
$$




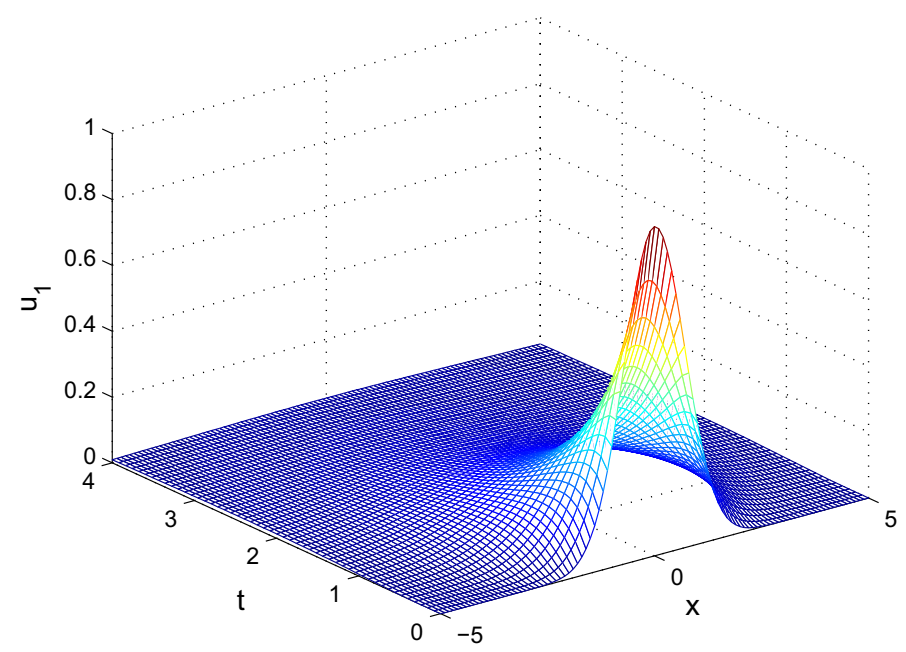

Fig. $13 u_{1}$

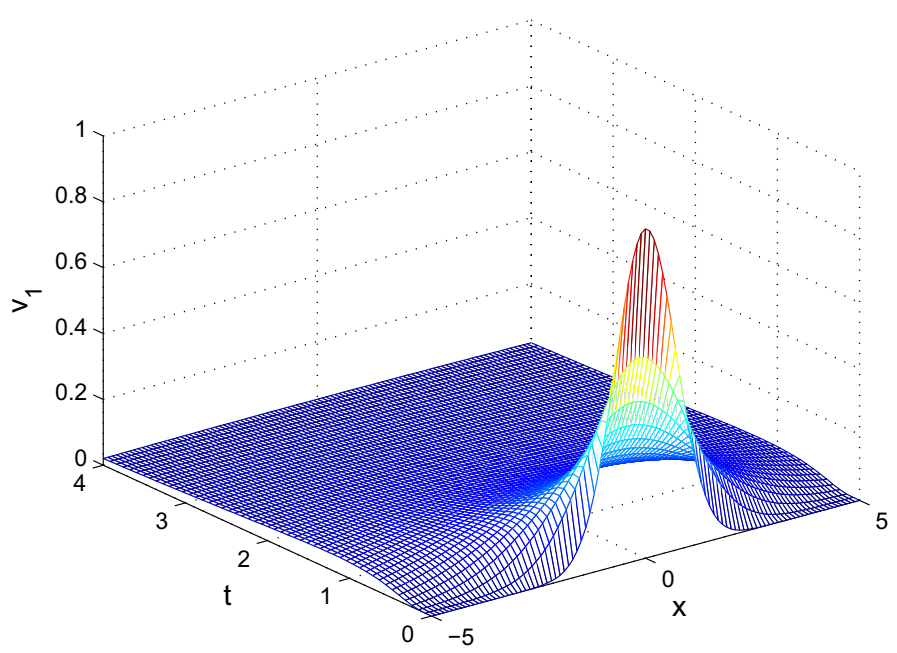

Fig. $14 v_{1}$

assuming an infinite domain with the decay conditions as $x \rightarrow \pm \infty$ at any finite time. The Eqs. (3.1)-(3.2) were solved using Fourier transforms in $x$.

Case $s=0, c=0, q_{1}=q_{2}=0.2, \mu_{1}=1, \mu_{2}=5$.

For small values of $q_{1}, q_{2}$, in the absence of shift, $s=0$, the system is in a stable equilibrium. Initial perturbations decay monotonically with the diffusion in $x$ adding to the widening of the solution shape in the $x$ direction (Figs. 13, 14).

Case $s=5, c=0, q_{1}=q_{2}=0.2, \mu_{1}=1, \mu_{2}=5$.

With $s=5$ and at higher values of $s$ illustrated in the subsequent diagrams, we begin to observe an initial transient spike in $u_{1}$ followed eventually by decay. There is little evident influence on the second component in the solution, $v_{1}$, leading us to 


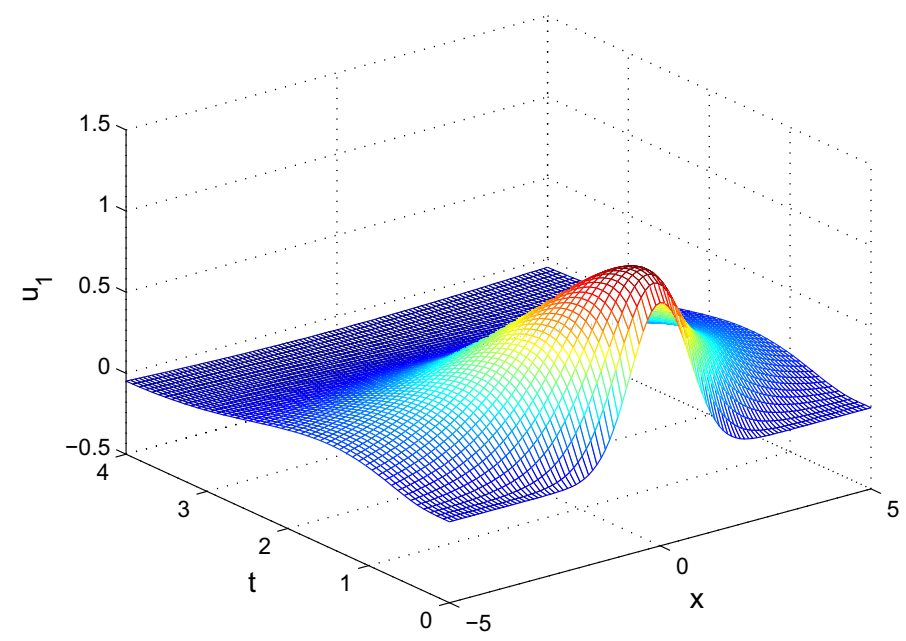

Fig. $15 u_{1}$

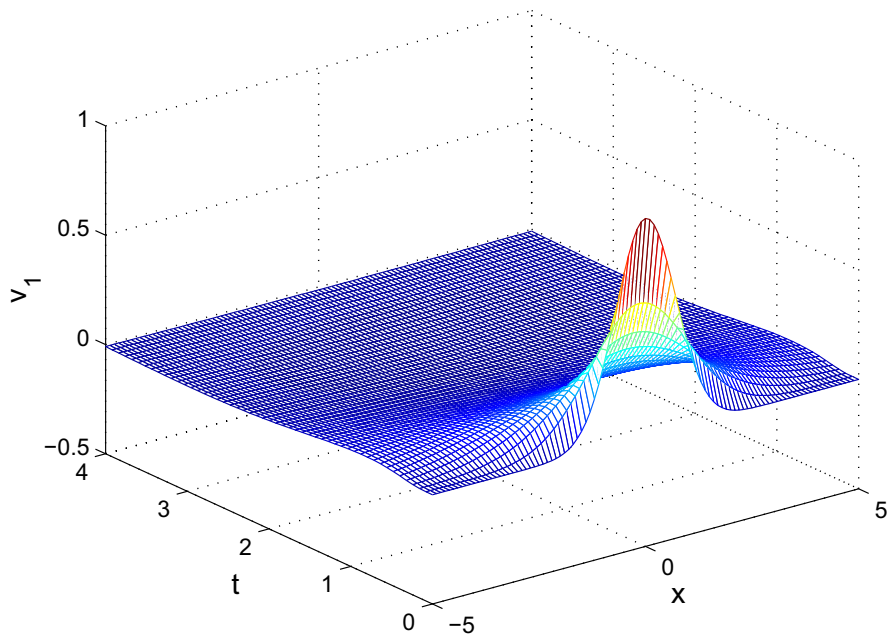

Fig. $16 v_{1}$

believe that the the second component acts as a background for the transient amplification of the overall perturbation level (Figs. 15, 16, 17, 18, 19).

Case $s=10, c=0, q_{1}=q_{2}=0.2, \mu_{1}=1, \mu_{2}=5$.

Case $s=25, c=0, q_{1}=q_{2}=0.2, \mu_{1}=1, \mu_{2}=5$.

The trend continues at higher values of $s$. As an alternative way to illustrate the effect of transient growth, here we show the norm of the solution,

$$
N(x, t)=\sqrt{u_{1}^{2}+v_{1}^{2}} .
$$

Case $s=10, c=1, q_{1}=q_{2}=0.2, \mu_{1}=1, \mu_{2}=5$. 


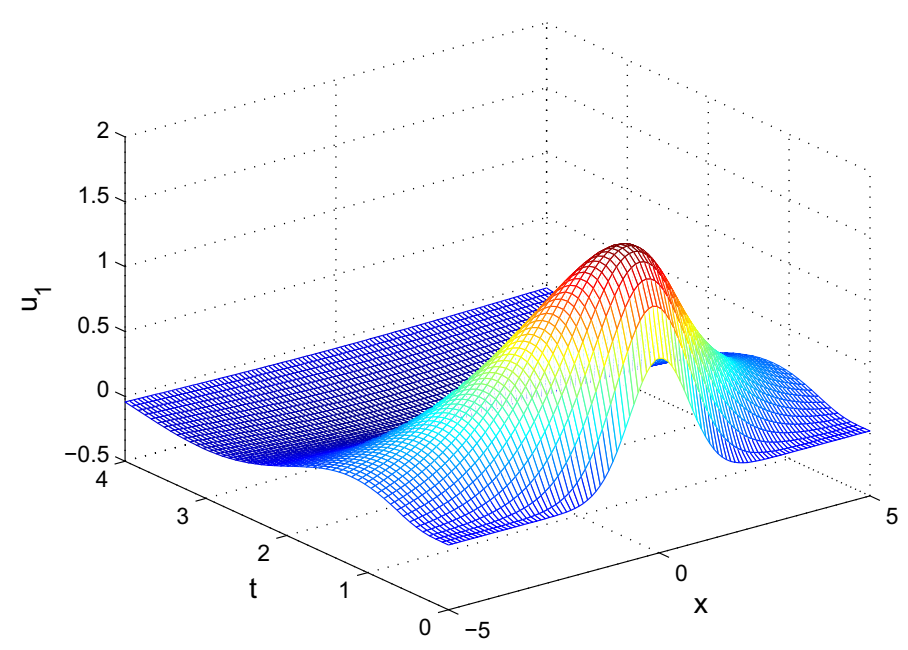

Fig. $17 u_{1}$

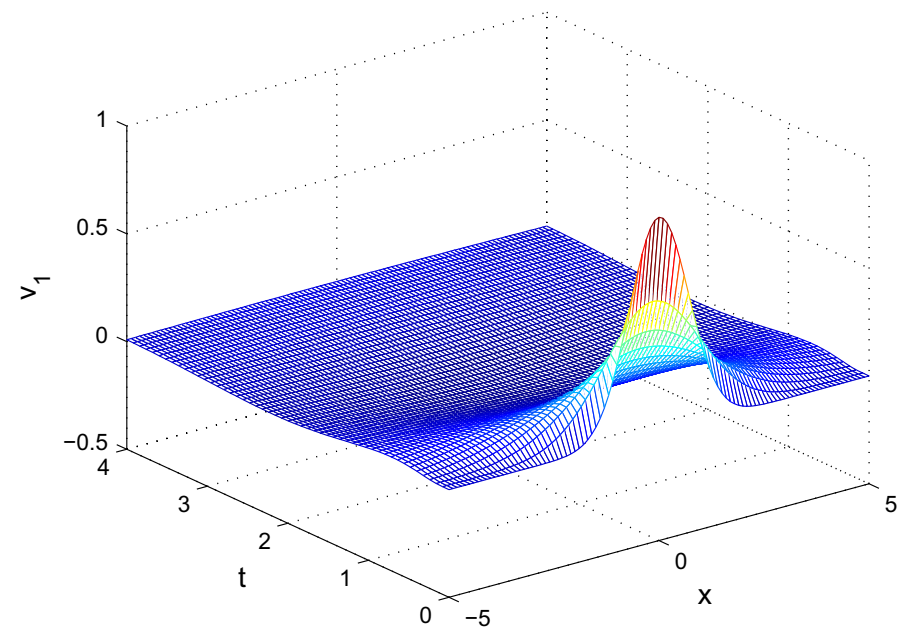

Fig. $18 v_{1}$

The contour plot in Fig. 20 gives a clear illustration of the effect of advection on the transient flow. The peak disturbance amplitude is achieved a little off centre, shifted in the direction of advection. A similar pattern was also observed in other computations for disturbances influenced by advection.

At sufficiently high values of $s$ the nature of the disturbance acquires features familiar from studies of convectively unstable systems. The maximum in the disturbance magnitude can be quite high, however the advective displacement of the most amplified part of the disturbance makes it less likely to be of significance in any finite-range system. Note however that in this study we do not evalute end effects as such. In theory 


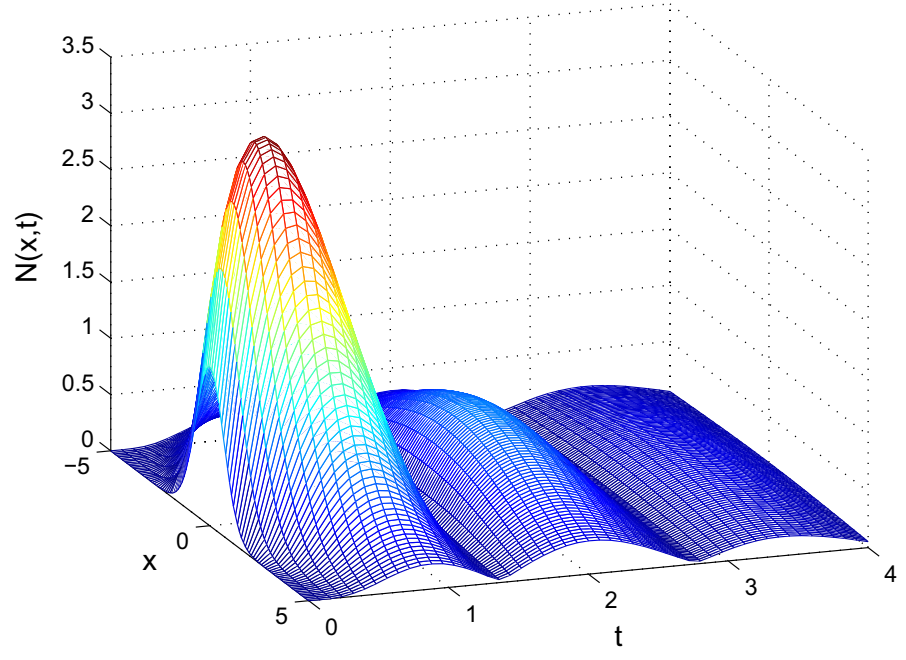

Fig. $19 N(x, t)$

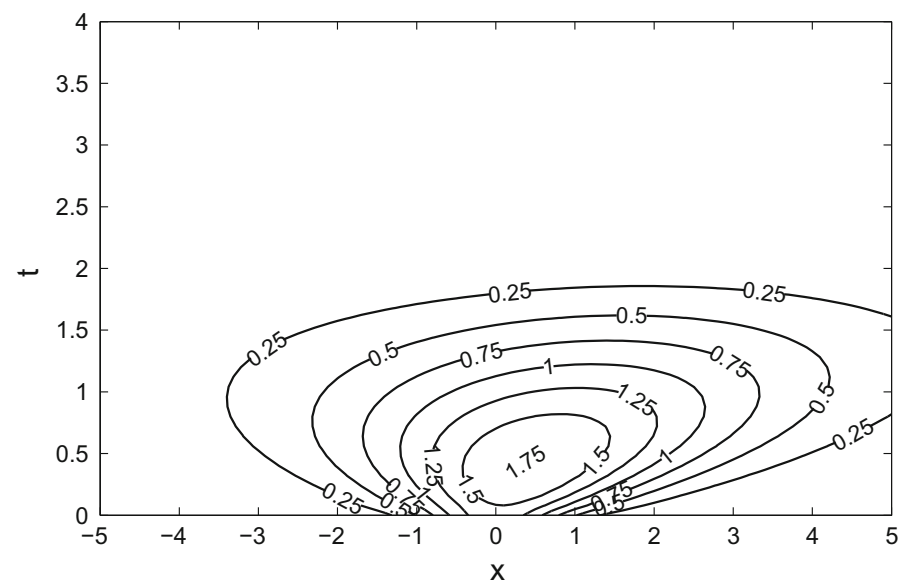

Fig. $20 N(x, t)$

at least, at the end points the perturbation may become entangled in a feedback loop providing further growth to the distrubance [10-12].

Case $s=10, c=0, q_{1}=1.7, q_{2}=0.2, \mu_{1}=1, \mu_{2}=5$.

Transient modes are interesting in the first place as potential triggers of non-linear behaviour. When sufficiently strong transients are present, they enter a competition with conventional long-time (asymptotic) instability. The illustration in the next figure shows how the two effects can co-exist. We observe a relatively mild instability at larger times which develops after an initial transient spike (Fig. 21). 


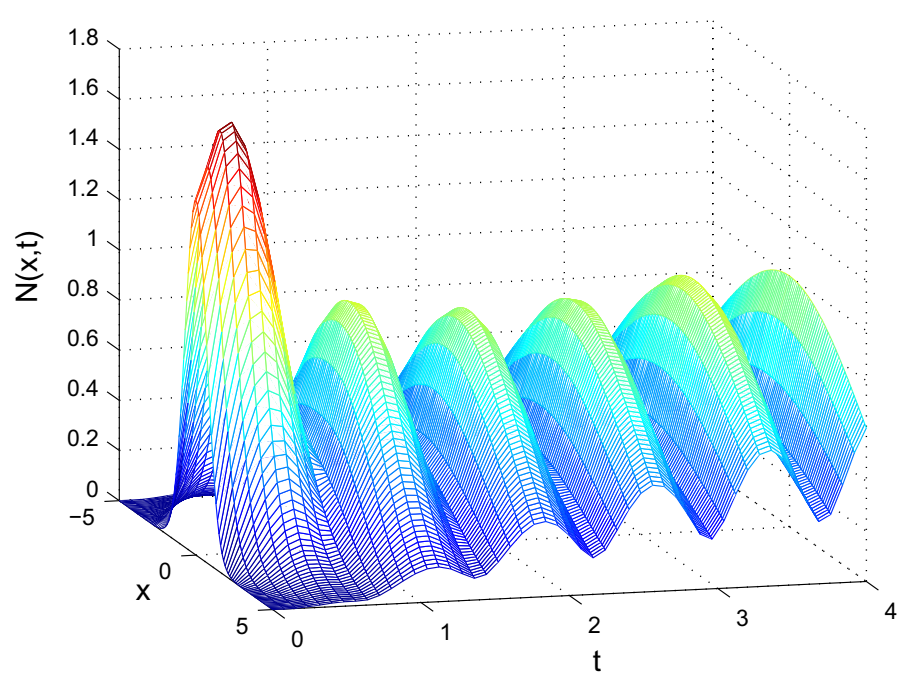

Fig. $21 N(x, t)$

\section{Conclusions}

In this study we have shown that transient effects appear in RDA systems under rather general conditions. Generally speaking, there is no direct correlation between transient growth and/or asymptotically stable or unstable behaviour at large times. Indeed, the extended Schnakenberg system exhibits transients of nearly equal strength in both asymptotically stable and unstable regimes illustrated in this research.

The original Schnakenberg model, proposed as an illustration of a tri-molecular autocatalitic reaction with complex non-linear behaviour, serves well in explaining non-trivial patterns such as oscillations, stripes and replicating spikes (e.g. [14]). The present study, devoted to early stages of the system evolution near a homogeneous equilibrium state, addresses a somewhat different aspect of the system dynamics with the emphasis on the non-monotonic amplification especially when the model is extended to account for possible stronger asymmetry of the kinetics. Clearly, direct comparisons with a realistic chemical (or any other) reactive setup would be premature at this stage, given the simplicity of the model. Rather the results of this study may become useful in interpreting a realistic system's behaviour prior to and during the start of non-linear evolution. Studies of this kind are understandably difficult.

The degree of transient amplification is a key to possible non-linear evolution scenarios in every particular system. From this point of view, a careful study of the parameter space is required in specific applications. For example, the conventional Schnakenberg model seems to predict limited transient growth, however with further asymmetry added to the kinetic matrix we observe enhanced transient amplification and a clear possibility of non-linear evolution initiated at early times, long before the asymptotic instability (when present) can initiate the conventional non-linear path. 
Acknowledgments This Project was funded by the Deanship of Scientific Research (DSR), King Abdulaziz University, Jeddah, under Grant No. 21/34/GR. The Authors, therefore, Acknowledge with thanks DSR Technical and financial support.

Open Access This article is distributed under the terms of the Creative Commons Attribution License which permits any use, distribution, and reproduction in any medium, provided the original author(s) and the source are credited.

\section{Appendix}

The initial conditions for (2.19)-(2.20) are found as follows. Let

$$
A=\left(\begin{array}{ll}
a_{11} & a_{12} \\
a_{21} & a_{22}
\end{array}\right)
$$

be matrix $A$ in (2.10) with the entries written symbolically. Then

$$
\left(\begin{array}{c}
U_{+} \\
V_{+}
\end{array}\right)=\left(\begin{array}{c}
f_{+} \\
1
\end{array}\right),\left(\begin{array}{l}
U_{-} \\
V_{-}
\end{array}\right)\left(\begin{array}{c}
f_{-} \\
1
\end{array}\right)
$$

with

$$
f_{+}=\frac{a_{12}}{\lambda_{+}-a_{11}}, f_{-}=\frac{a_{12}}{\lambda_{-}-a_{11}} .
$$

Then the eigenfunction coefficients in terms of the initial conditions are given by

$$
C_{2}=\frac{1}{2} \frac{u_{10}-v_{10} f_{+}}{f_{-}-f_{+}}, \quad C_{1}=\frac{v_{10}}{2}-\frac{1}{2} \frac{u_{10}-v_{10} f_{+}}{f_{-}-f_{+}}
$$

\section{References}

1. M.C. Cross, P.C. Hohenberg, Pattern formation outside of equilibrium. Rev. Mod. Phys. 65, 851-1112 (1993)

2. A. Elragig, S. Townley, A new necessary condition for Turing instability. Math. Biosci. 239, 131-138 (2012)

3. D.A. Garzon-Alvarado, C.H. Galeano, J.M. Mantilla, Turing pattern formation fro reactionconvection-diffusion systems in fixed domains submitted to toroidal velocity fields. Appl. Math. Model. 35, 4913-4925 (2011)

4. P. Liu, J. Shi, Y. Wang, X. Feng, Bifurcation analysis of reaction-diffusion Schnakenberg model. J. Math. Chem. 51, 2001-2019 (2013)

5. J.D. Murray, Mathematical biology: an introduction, vol. 1 (Springer, Berlin, 2002)

6. M.G. Neubert, H. Caswell, J.D. Murray, Transient dynamics and pattern formation: reactivity is necessary for Turing instabilities. Math. Biosci. 175, 1 (2002)

7. M.R. Ricard, S. Mischler, Turing instabilities at Hopf bifurcation. J. Nonlinear Sci. 19, 467-496 (2009)

8. L. Ridolfi, C. Camporeale, P. D'Odorico, F. Laio, Transient growth induces unexpected deterministic spatial patterns in the Turing process. EPL (Europhys. Lett.) 95, 18003 (2011)

9. J. Schnakenberg, Simple chemical reaction systems with limit cycle behaviour. J. Theor. Biol. 81, 389-400 (1979)

10. S.N. Timoshin, Feedback instability in a boundary-layer flow over roughness. Mathematika 52(1-2), 161-168 (2005) 
11. S.N. Timoshin, J.M. Linkins, Transient feedback and global instability in non-homogeneous systems. Philos. Trans. R. Soc. A Math. Phys. Eng. Sci. 363(1830), 1235-1245 (2005)

12. S.N. Timoshin, F.T. Smith, Non-local interactions and feedback instability in a high Reynolds number flow. Theor. Comput. Fluid Dyn. 17, 1-18 (2003)

13. A.M. Turing, The chemical basis of morphogenesis. Philos. Trans. R. Soc. Lond. Ser. B Biol. Sci. 237(641), 37 (1952)

14. M.J. Ward, J. Wei, The existence and stability of asymmetric spike patterns for the Schnackenberg model. Stud. Appl. Math. 109(3), 229-264 (2002) 